\title{
CHARACTERISATION OF MIXED NEUTRON-PHOTON WORKPLACE FIELDS AT NUCLEAR FACILITIES BY SPECTROMETRY (ENERGY AND DIRECTION) WITHIN THE EVIDOS PROJECT
}

\author{
M. Luszik-Bhadra ${ }^{1, *}$, D. Bartlett ${ }^{2}$, T. Bolognese-Milsztajn ${ }^{3}$, M. Boschung ${ }^{4}$, M. Coeck ${ }^{5}$, \\ G. Curzio ${ }^{6}$, F. d'Errico ${ }^{6}$, A. Fiechtner ${ }^{4}$, V. Lacoste ${ }^{3}$, L. Lindborg ${ }^{7}$, M. Reginatto', \\ H. Schuhmacher ${ }^{1}$, R. Tanner ${ }^{2}$ and F. Vanhavere ${ }^{5}$ \\ ${ }^{1}$ Physikalisch-Technische Bundesanstalt, Bundesallee 100, D-38116 Braunschweig, Germany \\ ${ }^{2}$ Radiation Protection Division, Health Protection Agency, Chilton, Didcot OX11 0RQ, UK \\ ${ }^{3}$ Institut de Radioprotection et de Sûreté Nucléaire, F-92265 Fontenay-aux-Roses, France \\ ${ }^{4}$ Paul Scherrer Institut, CH-5232 Villigen, Switzerland \\ ${ }^{5}$ Studiecentrum voor Kernenergie - Centre d'étude de l'énergie nucléaire, B-2400 Mol, Belgium \\ ${ }^{6}$ Dipartimento di Ingegneria Meccanica, Nucleare e della Produzione, I-56126 Pisa, Italy \\ ${ }^{7}$ Department of Medical Radiation Physics, Karolinska Institutet, SE 17176 Stockholm, Sweden
}

\begin{abstract}
Within the EC project EVIDOS, 17 different mixed neutron-photon workplace fields at nuclear facilities (boiling water reactor, pressurised water reactor, research reactor, fuel processing, storage of spent fuel) were characterised using conventional Bonner sphere spectrometry and newly developed direction spectrometers. The results of the analysis, using Bayesian parameter estimation methods and different unfolding codes, some of them especially adapted to simultaneously unfold energy and direction distributions of the neutron fluence, showed that neutron spectra differed strongly at the different places, both in energy and direction distribution. The implication of the results for the determination of reference values for radiation protection quantities (ambient dose equivalent, personal dose equivalent and effective dose) and the related uncertainties are discussed.
\end{abstract}

\section{INTRODUCTION}

The EVIDOS project funded by the European Commission within its fifth Framework Programme has been one of the largest projects in the last decade in the field of dosimetry and spectrometry of mixed neutron-photon workplace fields at nuclear facilities. It has been a cooperation of partners from seven institutions and seven countries within Europe. The name EVIDOS stands for 'EValuation of Individual DOSimetry in mixed neutron and photon radiation fields'. In order to evaluate the readings of radiation protection instruments - ambient dosemeters, passive personal dosemeters and new active electronic dosemeters - the reference values for radiation protection quantities needed to be determined by spectrometry.

This paper describes - after a short introduction of the workplace fields investigated - the methods used for determining energy and direction distributions of the neutron fluence, and deriving radiation protection quantities by folding these distributions with fluence-to-dose conversion coefficients, summarises spectra and radiation protection quantities as obtained in the places visited and discusses the uncertainties involved.

*Corresponding author: marlies.luszik-bhadra@ptb.de

\section{INVESTIGATED MIXED NEUTRON- PHOTON WORKPLACES}

In order to check the instrumentation and methods used, first measurements were performed in the wellcharacterised simulated workplace fields CANEL and SIGMA, provided by IRSN at Cadarache, France. This campaign was followed by measurements in 17 real workplace fields at nuclear facilities in Europe. The investigations comprised

- measurements inside two power reactors-a boiling water reactor in Krümmel, Germany and a pressurised water reactor in Ringhals, Sweden

- measurements at a research reactor-VENUS in Mol, Belgium,

- measurements at transport casks with spent fuel - one cask (type NTL11) provided without neutron transport shielding at the Krümmel nuclear power plant and one cask (Type TN 17/ MK 2) provided with a neutron transport shielding at the Ringhals nuclear power plant

- and measurements at fuel processing plants and storages-Belgonucléaire in Mol, Belgium and at a European nuclear facility (NF).

The measurement positions are described in more detail in Refs. 1 and 2. In general, two to four positions with different amounts of shielding were 
selected, and in all cases workplaces were chosen where neutrons contributed considerably $(23-96 \%)$ to the total dose equivalent.

\section{SPECTROMETERS FOR THE DETERMINATION OF ENERGY DISTRIBUTIONS}

The IRSN Bonner spheres system (Figure 1) has been used for the determination of neutron energy spectra. This spectrometer consists of 12 polyethylene spheres with diameters between 2.5 and 12 inches and a central ${ }^{3} \mathrm{He}$ thermal neutron counter (Type $0.5 \mathrm{NH} 1 / \mathrm{KI}$, manufactured by Eurisys Mesures). The five smaller spheres were used with and then without a $1 \mathrm{~mm}$ thick cadmium shield surrounding the detector. The response functions of this Bonner sphere spectrometer are well known. They have been calculated using the MCNP code, and the calculations were validated by results of measurements performed at PTB and NPL ${ }^{(3)}$.

The BTI ROSPEC spectrometer - a commercial spectrometer provided by Bubble Technology Industries - has been used in a few workplaces by IRSN. This spectrometer consists of a rotating platform with four spherical proportional counters for neutron measurement from $50 \mathrm{keV}$ to $4.5 \mathrm{MeV}$ and two ${ }^{3} \mathrm{He}$ detectors for the thermal and epithermal region. Unfortunately, it was not possible to derive reliable results using this spectrometer because of problems with noise, vibrations and not sufficiently well-known response functions.

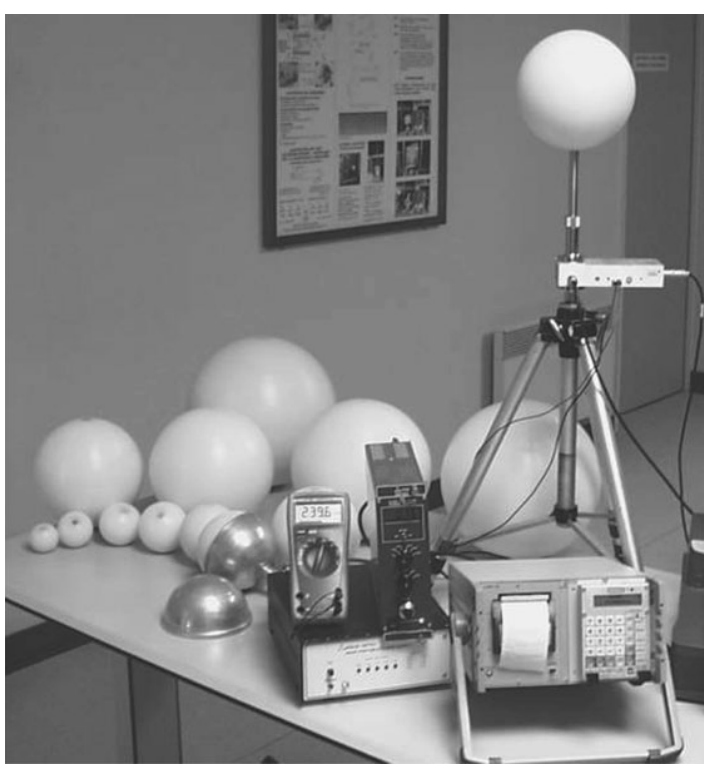

Figure 1. IRSN Bonner sphere spectrometer.

\section{ANALYSIS OF BONNER SPHERE DATA}

For reliable results using Bonner spheres, the response functions of the system should be well known and the analysis of the measurements should be performed using different unfolding codes, to cross-check solution spectrum. Within this project, a procedure has been worked out in collaboration between IRSN and PTB which was used in a systematic way for the analysis of all workplace fields and which resulted in especially small associated uncertainties. In the following, this method is described using as example the results obtained at a workplace at Belgonucléaire (position 2A, MOX fuel in a steel box).

Two unfolding codes developed at PTB were used.

- The NUBAY (Neutron spectrum Unfolding using BAYesian parameter estimation) $\operatorname{code}^{(4)}$ is a parameter estimation method. It assumes that the spectrum can be parameterised in an appropriate way using a finite set of parameters. For these parameters, posterior probability densities are provided by the code. In addition, it can provide probability densities for integral quantities, which is useful for estimating the integral quantities and their associated uncertainties.

- The GRAVEL code, a modification of SAND-II, is an iterative algorithm ${ }^{(5)}$. It provides a free form solution, which depends not on parameterisation, but which needs a starting spectrum (initial estimate).

For the analysis done with NUBAY, the neutron spectrum was approximated by a composite spectrum made up of three components: a thermal peak, a highenergy peak and an intermediate region. The thermal peak was modelled with a thermal Maxwellian spectrum $^{(6)}$, using two free parameters: one to describe the temperature and the other to describe the magnitude of the peak. The high-energy peak was modelled with a fission Maxwellian spectrum $^{(6)}$, also using two free parameters: one to describe the mean energy and the other to describe the magnitude of the peak. The intermediate region was modelled as a straight line in lethargy representation which went smoothly to zero at the low- and high-energy ends of the intermediate region, using at a minimum two free parameters: one to describe the slope of the intermediate region and the other to describe the magnitude of the intermediate region. In some cases, additional parameters were considered for the intermediate region, two to describe the locations of the low- and high-energy ends and two to describe the shape of the drop-offs at the low- and high-energy ends.

Figure 2 shows

- the resulting NUBAY solution spectra, which were obtained by using the mean values of the 

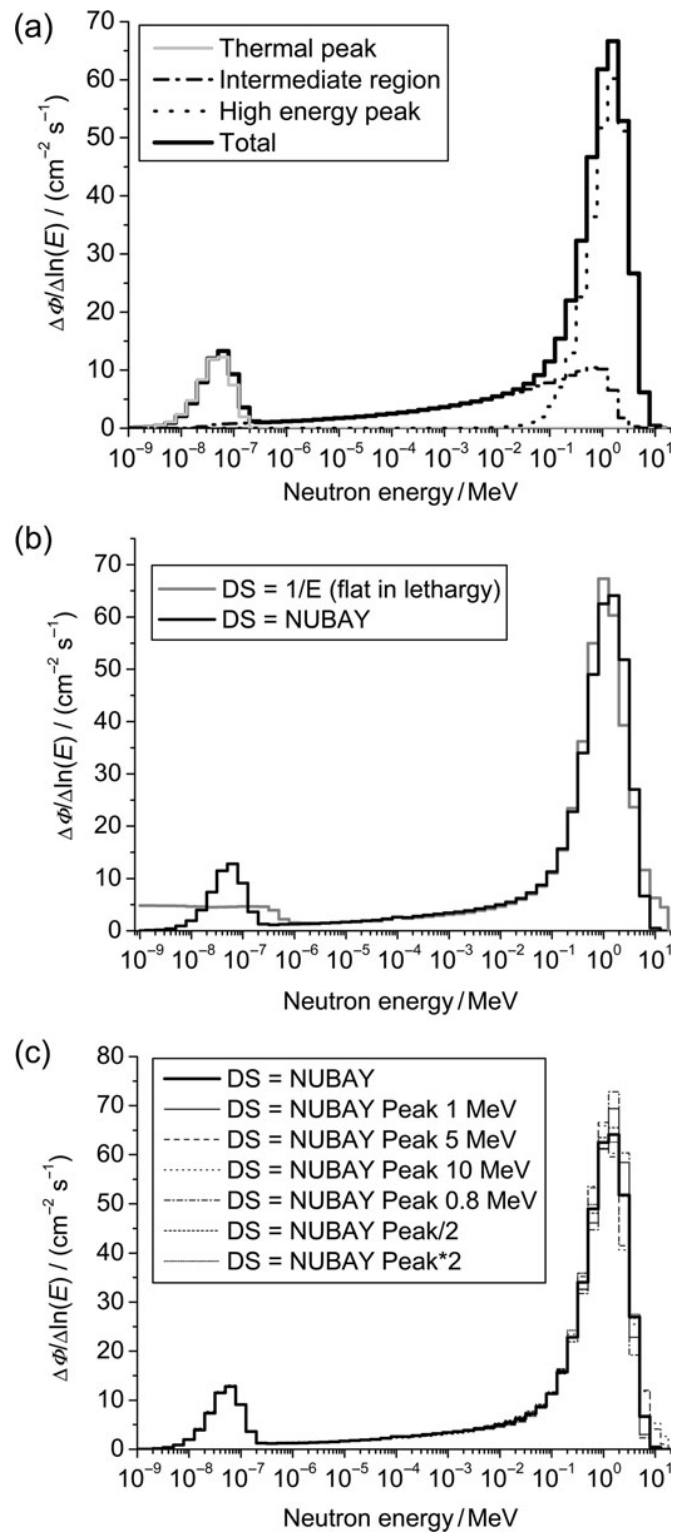

Figure 2. Resulting spectra as obtained by unfolding for the position 2A (MOX fuel in a steel box) at Belgonucléaire using the NUBAY code (a) and using the GRAVEL code $(b, c)$ with different initial spectra as indicated in the Figure inset.

parameters obtained by means of the NUBAY unfolding method,

- two GRAVEL solution spectra using as initial estimate the NUBAY solution spectrum and a spectrum flat in lethargy representation, respectively
- and different GRAVEL solution spectra, using as initial estimate the NUBAY solution spectrum and spectra in which the position and height of the high-energy peak of the NUBAY solution was varied.

Figure 2 shows that the resulting solution spectra are very similar. Only in the case of the GRAVEL unfolding with the spectrum flat in lethargy representation as initial estimate, the thermal peak was not resolved. The GRAVEL solution, taking the NUBAY solution spectrum as initial estimate, is finally considered as best solution.

Table 1 shows the integral fluence rates and ambient dose equivalent rates as derived from the different solution spectra as shown above. Standard deviations of the fluence and ambient dose equivalent rates, derived from the spread of the GRAVEL solution spectra were estimated and compared with the standard deviations computed by NUBAY, using Bayesian parameter estimation method. The standard deviations from both methods were consistent and as results, the solution neutron fluence energy distributions are the GRAVEL solution spectra using the NUBAY solution spectrum as prior information. In this case, a relative standard uncertainty of $0.6 \%$ resulted, both for the total fluence rate and for the ambient dose equivalent rate. The standard deviations from NUBAY and a 3\% systematic uncertainty from the response functions were added (quadratic sum) to calculate the total associated uncertainties. A similar high consistency was found for all spectra investigated at the different

Table 1. Integral fluence rates and ambient dose equivalent rates derived from the NUBAY and GRAVEL solutions and corresponding uncertainties (one standard deviation, see text).

\begin{tabular}{llll}
\hline Solution & \multicolumn{1}{c}{$\begin{array}{c}\text { Initial } \\
\text { estimate }\end{array}$} & $\begin{array}{c}\left(\mathrm{d} \Phi_{\text {tot }} / \mathrm{d} t\right) / \\
\left(\mathrm{cm}^{-2} \mathrm{~s}^{-1}\right)\end{array}$ & $\begin{array}{c}\left(\mathrm{d} H^{*}(10) / \mathrm{d} t\right) / \\
\left(\mu \mathrm{Sv} \mathrm{h} \mathrm{h}^{-1}\right)\end{array}$ \\
\hline NUBAY & $\begin{array}{l}\text { Parameterised } \\
\text { spectrum }\end{array}$ & $223.7 \pm 1.3$ & $207.5 \pm 1.2$ \\
GRAVEL & NUBAY & $223.0 \pm 1.4$ & $208.7 \pm 1.9$ \\
GRAVEL & Flat DS & 230.3 & 213.6 \\
GRAVEL & $\begin{array}{l}\text { NUBAY Peak } \\
\text { 1 MeV }\end{array}$ & 222.1 & 208.2 \\
GRAVEL & $\begin{array}{l}\text { NUBAY Peak } \\
\text { 5 MeV }\end{array}$ & 224.8 & 211.5 \\
GRAVEL & $\begin{array}{l}\text { NUBAY Peak } \\
\text { 10 MeV }\end{array}$ & 225.6 & 212.8 \\
GRAVEL & $\begin{array}{l}\text { NUBAY Peak } \\
\text { 0.8 MeV }\end{array}$ & 221.6 & 208.1 \\
GRAVEL & $\begin{array}{l}\text { NUBAY } \\
\text { Peak/2 }\end{array}$ & 223.1 & 208.3 \\
GRAVEL & $\begin{array}{l}\text { NUBAY } \\
\text { Peak*2 }\end{array}$ & 223.0 & 209.4 \\
& & & \\
\hline
\end{tabular}


workplaces. In all cases, it was possible to state values of total fluence rates and ambient dose equivalent rates with uncertainties $<5 \%(2,7-11)$.

Typical spectra measured in the workplaces and normalised to the corresponding total fluence are summarised in Figure 3. The spectra obtained at the power plants (BWR, PWR) showed a fast neutron peak at $\sim 100 \mathrm{keV}$ and considerable contributions in the thermal and intermediate energy region. At the research reactor (VENUS), the fast neutron peak was shifted to $\sim 1 \mathrm{MeV}$. The spectra obtained at the casks showed a fast neutron peak at $\sim 200 \mathrm{keV}$ and small contributions in the thermal and intermediate energy region for the cask without transport shielding (cask NTL) and larger contributions in the thermal and intermediate energy region for the cask with neutron transport shielding (cask TN). The spectra measured at Belgonucléaire $(\mathrm{BN})$ were quite hard spectra with a fast neutron peak at $\sim 1 \mathrm{MeV}$. The spectra measured at the NF were also hard spectra with a fast neutron peak at $\sim 1 \mathrm{MeV}$, but the shielding walls inside the storage facility produced considerable fluence contributions in the thermal and intermediate energy region.

Figure 4 shows the same spectra folded with fluence-to-ambient dose equivalent conversion coefficients. Since these coefficients increase for fast neutrons (by about a factor of 50), in all cases fast neutrons with energies above $50 \mathrm{keV}$ deliver the highest contribution to ambient dose equivalent. A good knowledge of the contributions in the thermal and intermediate energy regions is, however, still necessary, since several radiation protection instruments show high overreadings, especially in the intermediate energy region.

\section{SPECTROMETERS FOR THE DETERMINATION OF ENERGY AND DIRECTION DISTRIBUTIONS}

For the determination of energy and direction distributions of the neutron fluence, two newly developed spectrometers have been used.

One of them, developed at PTB, consists of a polyethylene sphere, $30 \mathrm{~cm}$ in diameter, and six capsules containing silicon detectors, mounted on the surface of this sphere (see Figure 5, left side). Each of the capsules contains four silicon detectors, covered with different neutron absorbers and converters. The pulse height spectra of all 24 detectors are recorded. The response of each detector depends on the energy and direction of the incoming neutron radiation. It is influenced by the different neutron absorbers and converters surrounding each detector, but also by the scattering, absorption and moderation of neutrons within the polyethylene sphere. The

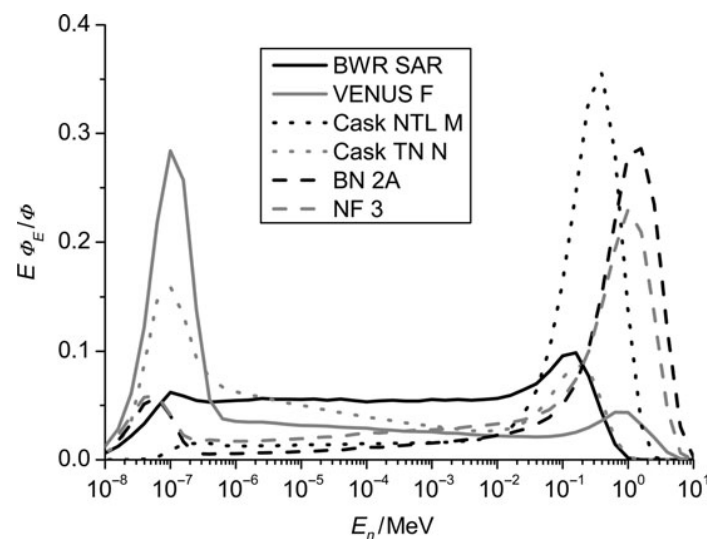

Figure 3. Energy distributions of neutron fluence determined within the EVIDOS project at workplaces (one significant spectrum per category)

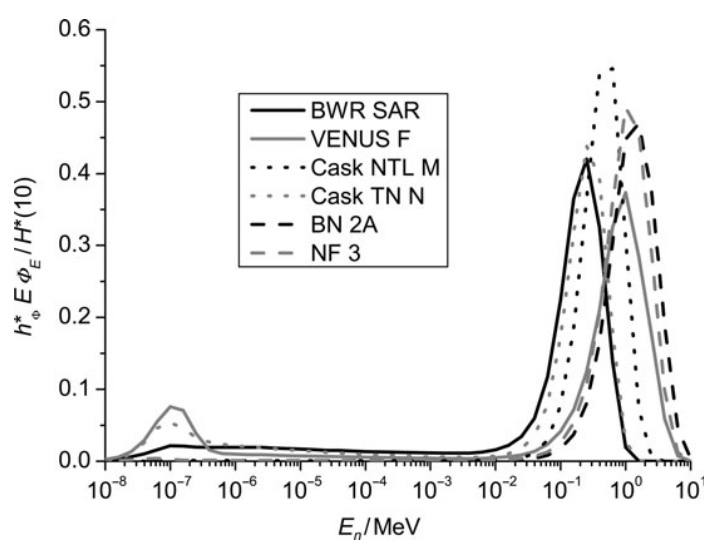

Figure 4. Energy distributions of neutron ambient dose equivalent determined within the EVIDOS project at workplaces (one significant spectrum per category).

response of the full system with all 24 detectors has been determined experimentally using irradiations with quasi-monoenergetic neutrons ranging from thermal up to $15 \mathrm{MeV}$ and has been calculated using MCNP in the intermediate neutron energy region. In addition, the response has been determined experimentally also with respect to photons with energies from $65 \mathrm{keV}$ to $7 \mathrm{MeV}$, in order to discriminate pulses at low pulse height induced by photon radiation ${ }^{(12)}$.

Another direction spectrometer (Figure 5, right side) has been developed at the DIMNP. It is based on a nylon sphere, also $30 \mathrm{~cm}$ in diameter, with a superheated drop detector in its centre and an opening viewing $1 / 6$ steradian. The signals are counted acoustically and the energy response of the 

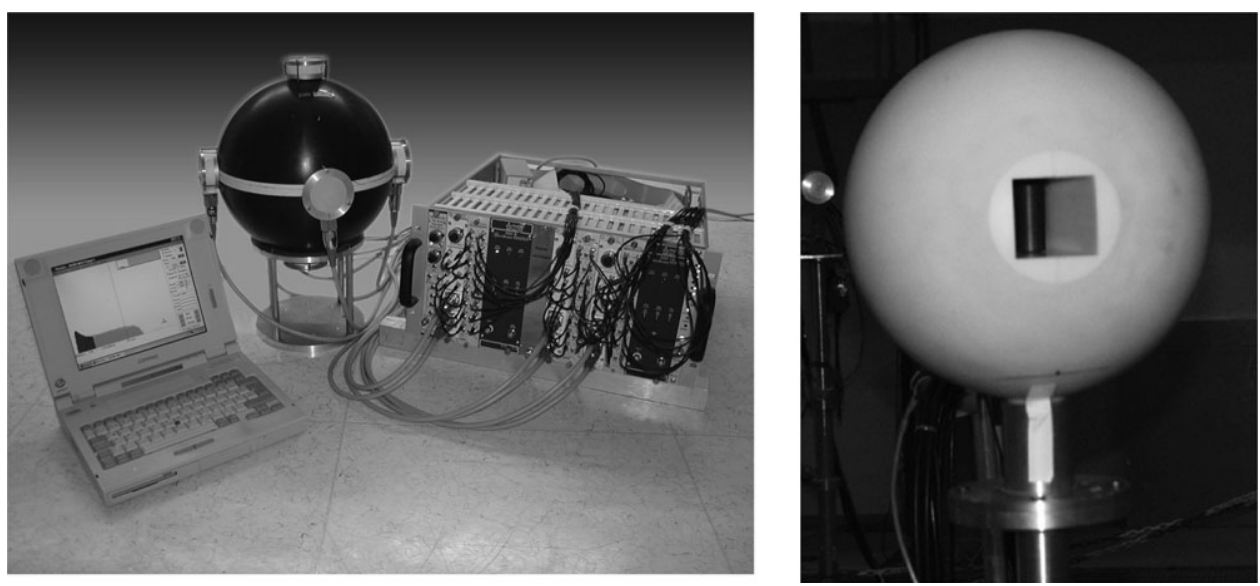

Figure 5. Directional spectrometer based on silicon detectors (on left side) and on a superheated drop detector (on the right side).

superheated drop detector is changed by varying its temperature between 30 and $55^{\circ} \mathrm{C}$. The response of this system has also been determined by measurements in mono-energetic neutron fields and by calculations using $\mathrm{MCNP}^{(13)}$. The response shows a threshold-like behaviour, both as function of energy and direction and is - in principle-well suited for the determination of energy and direction distributions of neutrons. However, operation of the spectrometer, which consisted of a series of successive temperature regulation, sphere rotation and bubble counting cycles, was highly time-consuming. Therefore, in none of the workplaces the full determination of energy and direction distributions of the neutron fluence could be achieved by this instrument.

\section{ANALYSIS OF DIRECTIONAL SPECTROMETER DATA}

For the analysis of the data provided by the directional spectrometer with silicon detectors, two different unfolding codes, both developed at PTB, were used.

- The MAXED unfolding code ${ }^{(14)}$ utilises maximum entropy combined neutron-photon-direction unfolding, which takes prior information into account in an optimised way.

- The MIEKE unfolding code ${ }^{(5)}$ uses maximum entropy to introduce a probability density. The original programme has been extended to perform also a combined neutron-photon-direction unfolding. It does not allow prior information to be used.

Since the response functions of the directional spectrometer with silicon detectors overlap in such a way that in general the direction distribution is not independent of the energy distribution, the best solution obtained was an unfolding using the MAXED code and prior information of energy distribution as was available from the Bonner sphere spectrometer.

But in order to estimate the uncertainties of the unfolding, in all cases four different unfolding calculations were performed.

- MAXED, BS: MAXED unfolding with prior information from Bonner sphere spectrometer,

- MAXED, 0.5 MeV: MAXED unfolding using a parameterised model spectrum assuming peaks at thermal and at $0.5 \mathrm{MeV}$ and an intermediate region that is flat in lethargy representation,

- MIEKE, $\mathrm{N}+\mathrm{G}$ : MIEKE unfolding, using full pulse height information of the silicon detectors and neutron-photon-direction unfolding,

- MIEKE, N: MIEKE unfolding, using only pulse height information above $800 \mathrm{keV}$ and neutrondirection unfolding (without photons, which contribute chiefly to the low pulse height signals).

Figure 6 shows, as an example, the result of the MAXED unfolding for position 2A at Belgonucléaire-MOX fuel in a steel box. The results of the unfolding are several spectra for different directional intervals. The direction of the intervals is indicated in the figure by FRONT (the assigned front direction was towards the steel box with MOX fuel), BACK, RIGHT, LEFT, UP and DOWN (defined as seen by a person looking towards the FRONT direction) and eight directions in between abbreviated by the first letter of the surrounding directions. In this case, the highest fluence was observed from FRONT direction and the integral energy distribution was close to that determined 


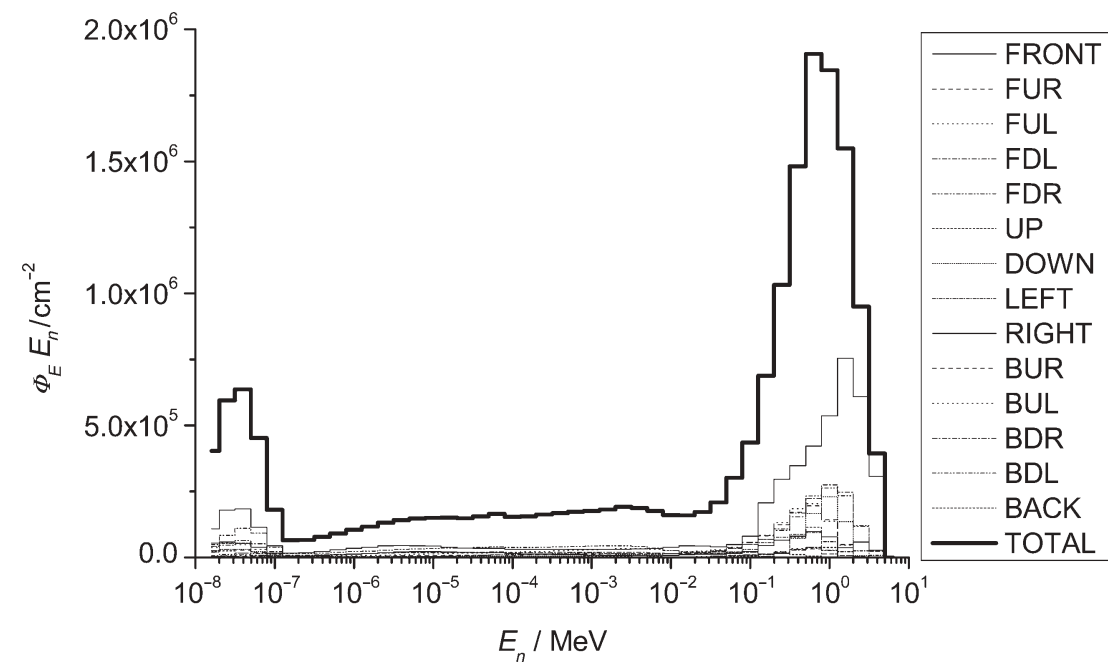

Figure 6. Energy distributions obtained for position 2A at Belgonucléaire for 14 directional intervals (see text).

by the Bonner sphere spectrometer (compare to results in Figure 2).

Using the full set of spectral distributions, integral values of ambient dose equivalent and personal dose equivalent were determined by folding the fluence distributions with conversion coefficients $h^{*}(10)$ and $h_{\mathrm{p}, \mathrm{slab}}(10)$, respectively, as given by ICRU $57^{(15)}$ and as derived for higher angles of incidence from recent calculations $^{(16)}$. For the calculations of the effective dose $E$, the fluences were folded using the conversion coefficients for AP, PA, LLAT and RLAT, and values for the directions 'Up' and 'Down' were estimated by setting them equal to ( $3 \cdot$ ISO-2.ROT). In addition, revised $\mathrm{w}_{\mathrm{R}}$ values - as recently proposed $^{(17)}$ - were also used to determine an effective dose $E_{\text {new. }}$ For this purpose, the fluence-to-dose conversion coefficients given in ICRU 57 for AP, PA, LLAT, RLAT, ISO and ROT were divided by the $\mathrm{w}_{\mathrm{R}}$ values as given in ICRU 57 and multiplied by the newly recommended values.

Figure 7 shows the relative contribution of neutrons from 14 directional intervals to $H^{*}(10)$ for the position $2 \mathrm{~A}$ at Belgonucléaire and also for a position inside the reactor containment at the Ringhals power plant (position PWR A). For the position 2A at Belgonucléaire, the highest contributions came from front, while inside the reactor containment, the distribution was much more isotropic. A more detailed presentation of the $H^{*}(10)$ distributions obtained in all workplace fields investigated within the EVIDOS project is given in Refs. 2 and 18.

While the fluence-to-ambient dose equivalent conversion coefficients do not depend on the direction of incidence, the fluence-to-personal dose equivalent coefficients decrease for low-energy neutrons (energy

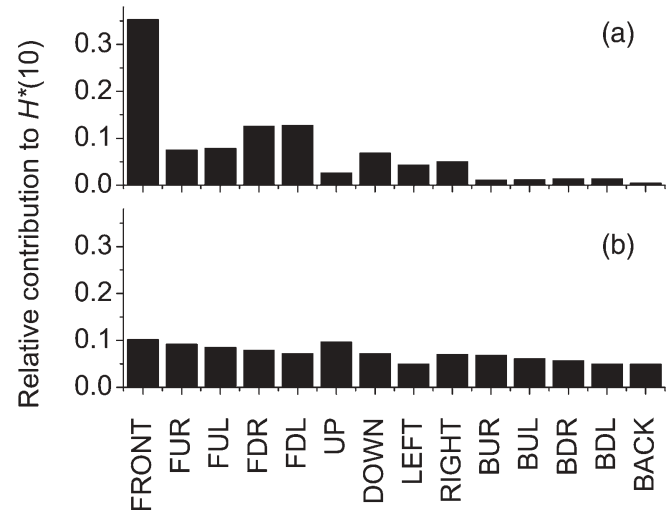

Figure 7. Relative contribution from 14 directional intervals to $H^{*}(10)$ for the position $2 \mathrm{~A}$ at Belgonucléaire (a) and position A at the Ringhals NPP (b).

below a few $\mathrm{MeV}$ ) at higher angles and are almost zero for neutrons impinging from the backward direction. Fluence-to-effective dose conversion coefficients are in general lower than fluence-to-ambient dose equivalent conversion coefficients for neutrons impinging at $0^{\circ}$, but change less as compared to the conversion coefficients for $H_{\mathrm{p}}(10)$ for neutrons impinging laterally $\left(90^{\circ}\right)$ or from backward direction $\left(180^{\circ}\right)$. This is illustrated in Figure 8 for neutrons with an energy of $200 \mathrm{keV}$.

This results in personal dose equivalent values close to the ambient dose equivalent in cases in which the radiation comes chiefly from front direction and in values roughly one third of the ambient dose equivalent for neutrons with isotropic 
incidence. In this way, the ratio $H_{\mathrm{p}}(10) / H^{*}(10)$ is a good indication of the directionality of the fields.

Figure 9 shows the ratio $H_{\mathrm{p}}(10) / H^{*}(10)$ as determined in all workplace fields investigated for the assigned FRONT direction, which was in most cases the direction with the highest contribution to dose equivalent. Especially low values of $H_{\mathrm{p}}(10) /$ $H^{*}(10)$ - in the order of 0.2 to 0.4 - were measured at positions with chiefly isotropic neutron distributions inside the power reactor containments (BWR SAR and PWR A), at a position close to the VENUS research reactor (VENUS F) and inside the highly shielded places at the NF (NF2, NF3). The lowest value $(0.22$, see MAXED, BS-unfolding result) was observed in the case of position BWR SAR, which was a position inside the fuel control

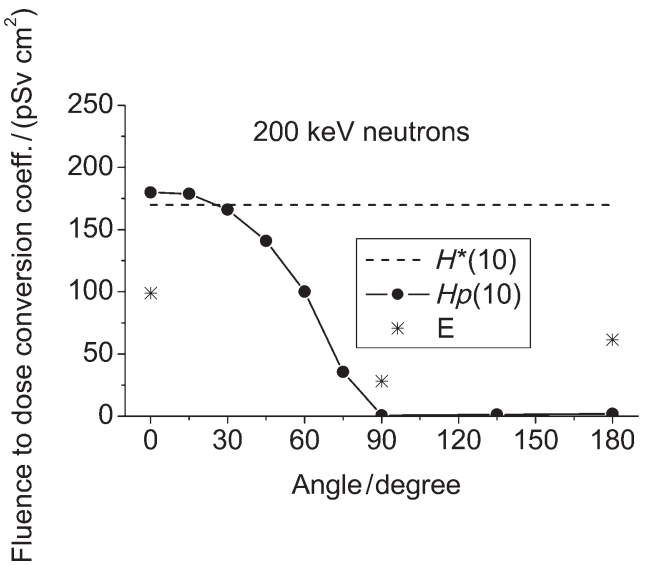

Figure 8. Fluence-to-dose conversion coefficients as function of direction ${ }^{(15,16)}$. In case of fluence-to-effective dose, the mean of the conversion coefficients LLAT and RLAT is shown at $90^{\circ}$. room below the reactor core at the Krümmel NPP. In this case, the highest contribution to dose equivalent was observed from the upwards direction and the assigned FRONT direction - which was, in this case, the direction towards the lock-did not coincide with the main dose direction. Higher values of $H_{\mathrm{p}}(10) / H^{*}(10)$ were observed at the simulated workplace fields CANEL and SIGMA, the casks and at Belgonucléaire. The highest value in workplaces at nuclear facilities was found for MOX fuel in a steel box in position $2 \mathrm{~A}$ at Belgonucléaire $(0.78$, see MAXED, BS-unfolding result). In Figure 9, the values determined by different unfolding procedures are indicated. In most cases, the values agree within $10 \%$, but in some cases also larger deviations $(\sim 30 \%)$ were observed. These large deviations are partly due to very limited statistics because of limited time available. For example, the measurement at the cask in Krümmel at the side position (cask NTL S) was performed in roughly one hour with an ambient dose equivalent rate of $55 \mu \mathrm{Sv} \mathrm{h}^{-1}$. Thus, energy and direction distribution of fluence was determined for a total ambient dose equivalent of $66 \mu \mathrm{Sv}$, a dose which is below the detection limit of several personal dosemeters. The standard uncertainty assigned to the ratio $H_{\mathrm{p}}(10) / H^{*}(10)$ as determined in all workplace fields investigated for the assigned FRONT direction was estimated conservatively as $30 \%$.

For the determination of reference values $H_{\mathrm{p}}(10)$, the best estimates as derived for $H_{\mathrm{p}}(10) / H^{*}(10)$ from the results of the directional spectrometer using the MAXED unfolding were taken and multiplied with values of $H^{*}(10)$ as obtained from the measurements with the Bonner sphere spectrometer. Final reference values, which were used for the determination of the response of personal dosemeters, are given in Refs. 2 and 18.

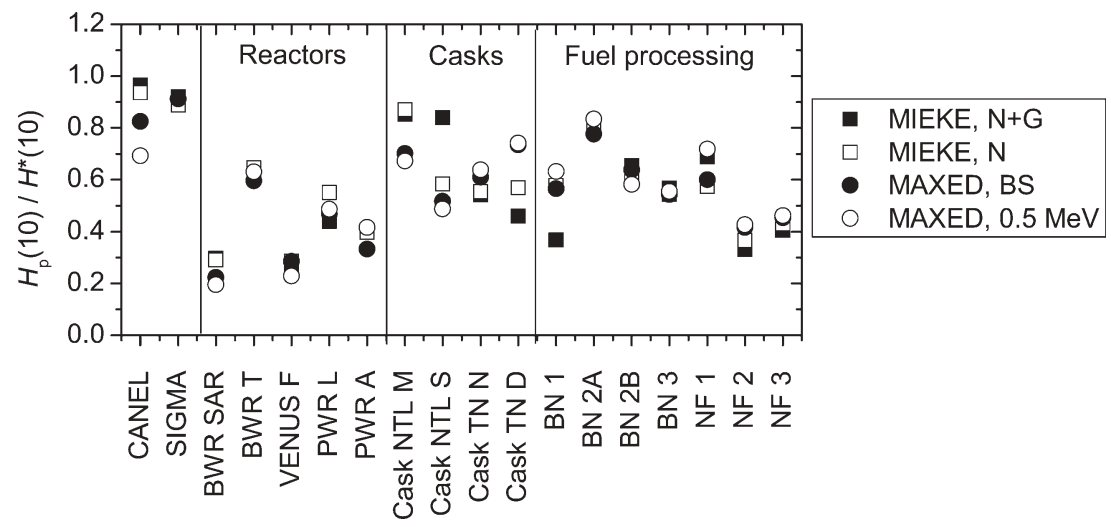

Figure 9. Ratio $H_{\mathrm{p}}(10) / H^{*}(10)$ as determined in all workplace fields investigated for the assigned FRONT direction and different unfolding methods (see text). 


\section{LUSZIK-BHADRA ET AL.}

Here, additional information is shown for radiation protection quantities (personal dose equivalent $H_{\mathrm{p}}(10)$ and effective dose $E$ ) for different orientation of a phantom or person in the fields investigated.

Figure 10 shows values $H_{\mathrm{p}}(10) / H^{*}(10)$ for the FRONT direction (as defined above) and for a phantom facing the afore-assigned LEFT, RIGHT, BACK directions and directions at the top and bottom. Strong variations are observed in fields where neutrons have come chiefly from front direction. In these cases, $H_{\mathrm{p}}(10)$ is close to $H^{*}(10)$ for the FRONT direction, but drastically smaller for the BACK direction. In case of the more isotropic fields (reactor fields BWR SAR, PWR A, VENUS F, NF 2, NF 3) the values scatter much less.

Figure 11 shows corresponding values for $E / H^{*}(10)$. These values scatter much less than the corresponding values $H_{\mathrm{p}}(10) / H^{*}(10)$ in all cases.

Table 2 shows values $E / H_{\mathrm{p}}(10)$ and $\boldsymbol{E}_{\text {new }} / H_{\mathrm{p}}(10)$ for the assigned FRONT direction. In most cases, $H_{\mathrm{p}}(10)$ is a conservative estimate of $E$. Exceptions with slightly higher $E$ values are the reactor fields BWR SAR, PWR A and VENUS F, where higher dose contributions were observed from upward directions, either caused by the reactor being above or by skyshine neutrons. $H_{\mathrm{p}}(10)$ is in all cases a conservative estimate of $\boldsymbol{E}_{\text {new }}$, since the change of conversion factors has, strictly speaking, the strongest influence on effective dose values for power reactors (see last column in Table 2).

\section{UNCERTAINTIES}

The question of reliability of data is important and not easy to answer in the case of complex instrumentation and unfolding methods.
Within the EVIDOS campaign, an especially low uncertainty has been stated for the determination of $H^{*}(10)$ using Bonner sphere spectrometry. This uncertainty $(<5 \%)$ is much lower than that given $15 \mathrm{y}$ ago in a measurement campaign using different spectrometers, also performed at the Ringhals NPP $(\sim 15 \%)^{(19,20)}$. In addition, new directional spectrometry, which has not been used in this way before, has been applied to determine $H_{\mathrm{p}}(10) /$ $H^{*}(10)$ values.

In order to check if the absolute uncertainties given are reliable, results of new measurements can be compared with those of the previous campaign ${ }^{(19,20)}$ and with results of calculations ${ }^{(21-23)}$, as far as available. The data are, however, very rare.

The previous measurement campaign resulted in $\mathrm{d} H^{*}(10) / \mathrm{d} t$ values 1820 and $280 \mu \mathrm{Sv} \mathrm{h}^{-1}$ for PWR A (containment) and PWR L (lock), respectively, which has to be compared with the results of this campaign (1850 and $253 \mu \mathrm{Sv} \mathrm{h}^{-1}$ ). While the values for PWR A agree well, those for PWR L differ by $\sim 10 \%$. However, it is not clear if these changes may be due to a different burn up of fuel in 1992 and 2004. It is mentioned that in both campaigns recoil proton spectrometry, which in principle can deliver higher resolution neutron spectra, did not work sufficiently well. Although the unfolding of Bonner sphere readings gives, in general, quite stable values for integral fluences, higher resolution spectrometry may change dose equivalent values, since the spectra at power plants and at casks with used fuel are peaked just in a region where fluence-to-dose conversion coefficients change drastically.

MCNP calculations have been performed for the simulated workplace fields CANEL and SIGMA. While the calculated value for SIGMA $\left(\mathrm{d} H^{*}(10) / \mathrm{d} t=\right.$ $\left.144 \mu \mathrm{Sv} \mathrm{h}^{-1}\right)^{(21)}$ agreed well with the measured one

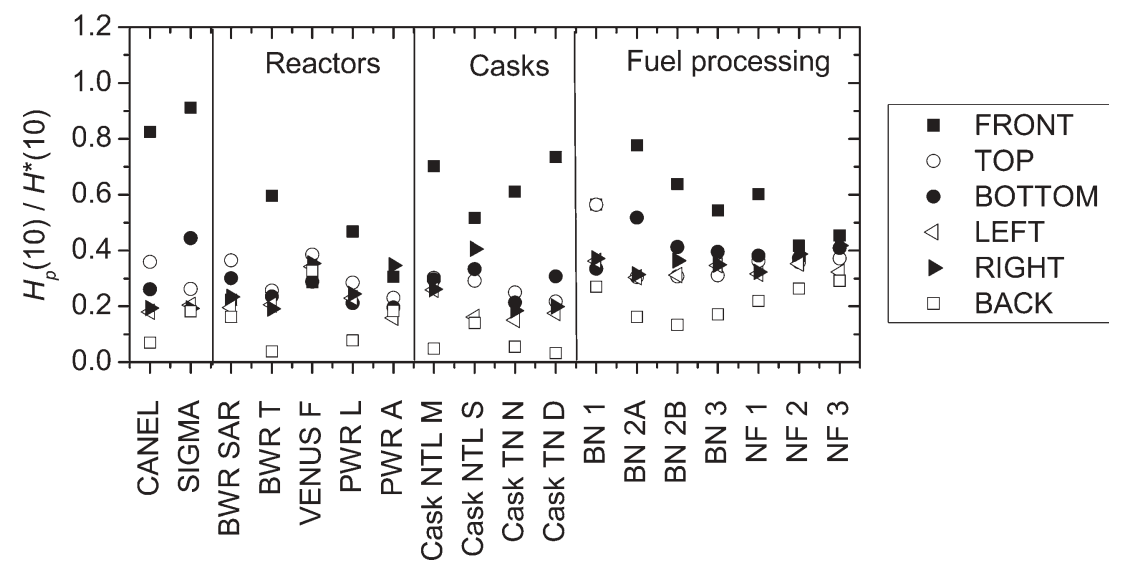

Figure 10. Ratio $H_{\mathrm{p}}(10) / H^{*}(10)$ as determined in all workplace fields investigated for different orientation of the phantom (see text). 


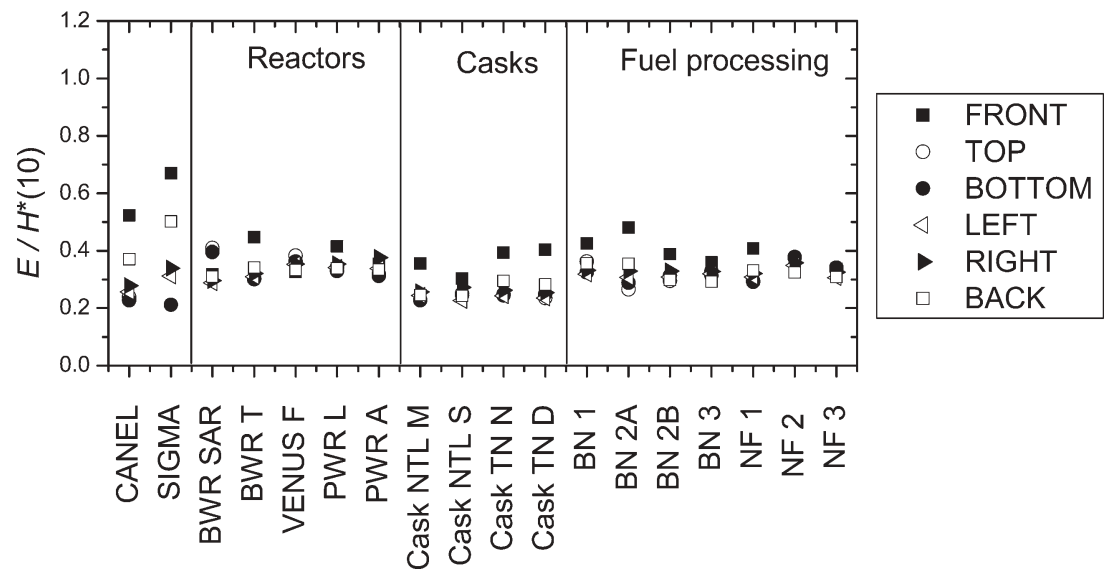

Figure 11. Ratio $E / H^{*}(10)$ as determined in all workplace fields investigated for different orientation of the person (see text).

Table 2. Values of $E / H_{\mathrm{p}}(10), E_{\text {new }} / H_{\mathrm{p}}(10)$ and $E_{\text {new }} / E$ for the assigned FRONT direction.

\begin{tabular}{lccc}
\hline Measurement position & $E / H_{\mathrm{p}}(10)$ & $E_{\text {new }} / H_{\mathrm{p}}(10)$ & $E_{\text {new }} / E$ \\
\hline CANEL & 0.63 & 0.47 & 0.75 \\
SIGMA & 0.73 & 0.56 & 0.77 \\
BWR SAR & 1.42 & 0.82 & 0.58 \\
BWR T & 0.75 & 0.45 & 0.60 \\
VENUS F & 1.14 & 0.89 & 0.79 \\
PWR L & 0.89 & 0.52 & 0.58 \\
PWR A & 1.16 & 0.64 & 0.56 \\
Cask NTL M & 0.51 & 0.43 & 0.85 \\
Cask NTL S & 0.59 & 0.49 & 0.84 \\
Cask TN N & 0.64 & 0.40 & 0.63 \\
Cask TN D & 0.55 & 0.39 & 0.71 \\
BN 1 & 0.75 & 0.73 & 0.97 \\
BN 2A & 0.62 & 0.60 & 0.96 \\
BN 2B & 0.61 & 0.55 & 0.91 \\
BN 3 & 0.66 & 0.58 & 0.88 \\
NF 1 & 0.68 & 0.62 & 0.91 \\
NF 2 & 0.84 & 0.71 & 0.85 \\
NF 3 & 0.74 & 0.70 & 0.94 \\
\hline
\end{tabular}

$\left(147 \mu \mathrm{Sv} \mathrm{h}^{-1}\right)$, there have been larger discrepancies of $\sim 10 \%$ for the CANEL facility (111 pSv per monitor count calculated ${ }^{(22)}, 122 \mu \mathrm{Sv}$ per monitor count measured). These discrepancies were recently solved when it was found that the water content in the moderator used was different from that assumed before, so that now calculations agree in an excellent way with the results of measurements $(122 \mathrm{pSv}$ per monitor count calculated) ${ }^{(23)}$.

This discussion shows that there is not a clear answer, at the moment, to the uncertainty which can be assigned to $H^{*}(10)$ values measured at nuclear facilities. But the problems involved indicate that a slightly more conservative estimate $(5-10 \%)$ may be realistic.

Values of $H_{\mathrm{p}}(10) / H^{*}(10)$ have also been determined in the previous campaign, however by using a simplified method. It has been assumed that the field is composed of an isotropic and a directed part and that both components can be estimated using the readings of TLD-Albedo and track detectors. Despite the simpler technique, results obtained earlier at PWR A and PWR L (0.41 and 0.42) $)^{(19)}$ agree sufficiently well-within $20 \%$-with the new values (0.33 and 0.47). Higher discrepancies (33\%) were found for previous and new measurements at a cask (previous: 0.49; now: 0.73). MCNP calculations of $H_{\mathrm{p}}(10) / H^{*}(10)$ have been performed for SIGMA, CANEL and VENUS $F$. The calculated values $(0.98,0.92,0.24)$ agree within $20 \%$ with the values determined now $(0.83,0.91,0.29)$. The agreement is in most cases within the uncertainties estimated $(30 \%)$.

\section{SUMMARY}

In total, 17 mixed neutron-photon workplaces were characterised within the EVIDOS project by neutron spectrometry at nuclear facilities. According to the results of the Bonner sphere spectrometer, the examined workplace spectra showed large difference in terms of thermal, epithermal and fast neutron components. The Bonner sphere spectrometer has shown high performance and can be considered to be a well-established reference instrument with low uncertainties for the determination of reference values $H^{*}(10)$.

Compared to this highly established method, directional spectrometers are still considered to be 


\section{LUSZIK-BHADRA ET AL.}

research instruments with much higher uncertainties - in the order of $30 \%$-yet involved. The direction distributions obtained showed considerable variations ranging from isotropic distributions to more directed ones. These different direction distributions had a strong influence on the personal dose equivalent reference values $H_{\mathrm{p}}(10)$, which were up to almost a factor of 5 lower than corresponding values of ambient dose equivalent $H^{*}(10)$. Ratios $H_{\mathrm{p}}(10) /$ $H^{*}(10)$ varied between 0.22 and 0.78 . The lowest value has been observed inside the control rod room below the core of the Krümmel NPP. $H_{\mathrm{p}}(10)$ values were found to be conservative estimates of the effective dose values $E$ for the assigned front direction in cases where this direction coincided with the main dose direction.

\section{ACKNOWLEDGEMENTS}

The authors wish to express their gratitude to the staffs at Cadarache, Krümmel, Mol and Ringhals who helped during the campaigns.

\section{FUNDING}

This research is partly funded by the European Commission under the auspices of the Euratom 5th Framework Programme: Nuclear Energy, 19982002, Contract No. FIKR-CT-2001-00175.

\section{REFERENCES}

1. d'Errico, F., Bartlett, D., Bolognese-Milsztajn, T., Boschung, M., Coeck, M., Curzio, G., Fiechiner, A., Kyllonen, J.-E., Lacoste, V., Lindborg, L., LuszikBhadra, M., Reginatto, M., Schuhmacher, H., Tanner, R. and Vanhavere, F. Evaluation of individual dosimetry in mixed neutron and photon radiation fields (EVIDOS). Part I: scope and methods of the project. Radiat. Prot. Dosim., doi: 10.1093/rpd/ncm169 (2007).

2. Schuhmacher, H. Evaluation of individual dosimetry in mixed neutron and photon radiation fields. PTB report PTB-N49 (2006).

3. Lacoste, V., Gressier, V., Pochat, J.-L., Fernández, F., Bakali, M. and Bouassoule, T. Characterization of Bonner sphere systems at mono-energetic and thermal neutrons fields, Radiat. Prot. Dosim. 110(1-4), 529-532 (2004).

4. Reginatto, M. Bayesian approach for quantifying the uncertainty of neutron doses derived from spectrometric measurements. Radiat. Prot. Dosim. 120, 64-69 (2006).

5. Matzke, M. Unfolding of pulse height spectra: the HEPRO program system. PTB Report PTB-N-19 (Braunschweig: PTB) (1994).

6. International Atomic Energy Agency. Neutron fluence measurements. Technical Reports Series No. 107 (Vienna: IAEA) (1970).
7. Lacoste, V., Reginatto, M., Assélineau, B. and Muller, H. Bonner sphere neutron spectrometry at nuclear workplaces in the framework of the EVIDOS project. Radiat. Prot. Dosim., doi: 10.1093/rpd/ncm161 (2007).

8. Lacoste, V., Assélineau, B. and Muller, H. Neutron spectrometry with Bonner spheres at workplaces in nuclear facilities at Krümmel (Germany) - EVIDOS Campaign no 1, IRSN Report-DRPH/SDE/2005-04 (2005).

9. Lacoste, V., Reginatto, M. and Muller, H. Neutron spectrometry with Bonner spheres at the Belgonucléaire MOX fuel processing plant - EVIDOS Campaign no. 2, IRSN Report DRPH/SDE/2004-34 (2004).

10. Lacoste, V. and Muller, H. Neutron spectrometry with Bonner spheres at the VENUS research reactorEVIDOS Campaign no 2. IRSN Report-DRPH/ SDE/2004-56 (2004).

11. Lacoste, V., Assélineau, B. and Muller, H., Neutron spectrometry with Bonner spheres at workplaces in nuclear facilities at Ringhals (Sweden)-EVIDOS Campaign no 3, IRSN Report - DRPH/SDE/2005-02 (2005).

12. Luszik-Bhadra, M., Reginatto, M. and Lacoste, V. Measurement of energy and direction distribution of neutron and photon fluences in workplace fields. Radiat. Prot. Dosim. 110(1-4), 237-241 (2004).

13. d'Errico, F., Giusti, V., Reginatto, M. and Wiegel, B. A telescope-design directional neutron spectrometer. Radiat. Prot. Dosim. 110(1-4), 533-537 (2004).

14. Reginatto, M., Luszik-Bhadra, M. and d'Errico, F. An unfolding method for directional spectrometers. Radiat. Prot. Dosim. 110(1-4), 539-543 (2004).

15. International Commission on Radiation Units and Measurements. Conversion coefficients for use in radiological protection against external radiation. ICRU Report 57 (Bethesda, MD: ICRU) (1998).

16. d'Errico, F., Giusti, V. and Siebert, B. R. L. A new neutron monitor and extended conversion coefficients for $H_{p}(10)$. To be published.

17. Dietze, G. and Harder, D. Proposal for a modified radiation weighting factor for neutrons. In: 11th International Congress of the International Radiation Protection Association, 23-28 May 2004 (Madrid, Spain. Published on CD).

18. Luszik-Bhadra, M., Bolognese-Milsztajn, T., Boschung, M., Coeck, M., Curzio, G., d'Errico, F., Fiechtner, A., Lacoste, V., Lindborg, L., Reginatto, M., Schuhmacher, H., Tanner, R. and Vanhavere, F. Direction distributions of neutrons and reference values of the personal dose equivalent in workplace fields. Radiat. Prot. Dosim., doi: 10.1093/rpd/ncm189 (2007).

19. Bartlett, D., Drake, P., Lindborg, L., Klein, H., Schmitz, Th. and Tichy, M. Determination of the neutron and photon dose equivalent at work places in nuclear facilities of Sweden. An SSI - EURADOS comparison exercise. Part 2: evaluation, Swedish Radiation Protection Authority, SSI Report 99:13 (1999).

20. Lindborg, L., Bartlett, D., Drake, P., Klein, H., Schmitz, Th. and Tichy, M. Determination of neutron and photon dose equivalent at work places in nuclear facilities in Sweden: a joint SSI - EURADOS comparison exercise. Radiat. Prot. Dosim. 61(1-3), 89-100 (1995). 


\section{CHARACTERISATION OF WORKPLACE FIELDS BY SPECTROMETRY}

21. Lacoste, V., Gressier, V., Muller, H. and Lebreton, L. Characterisation of the IRSN graphite moderated Americium-Beryllium neutron field. Radiat. Prot. Dosim. 110(1-4), 135-139 (2004).

22. Lacoste, V. and Gressier, V. Monte Carlo simulation of the IRSN CANEL/T400 realistic mixed neutron/ photon radiation field. Radiat. Prot. Dosim. 110(1-4), 123-127 (2004).

23. Lacoste, V. and Gressier, V. Monte Carlo simulation of the operational quantities at the realistic mixed neutronphoton radiation fields CANEL and SIGMA. Radiat. Prot. Dosim., doi: 10.1093/rpd/ncm202 (2007). 